\title{
Comparative Study on Overturning Moment and Eccentricity of A I-Shaped Building by Equivalent Lateral Force and Modal Response Spectrum Method Using ETABS Software M. T. Hasan*1, M. L. Hossain² \\ ${ }^{*}$ Workshop Maintenance Engineer, Department of Civil Engineering, Sylhet Engineering College, Sylhet, Bangladesh
}

${ }^{2}$ Structural Design Engineer, Bismillah Engineering and consultancy, Sirajganj, Bangladesh

\begin{abstract}
Article Info

Volume 8, Issue 4

Page Number : 173-179

\section{Publication Issue}

July-August-2021

\section{Article History}

Accepted : 02 July 2021

Published : 09 July 2021

Extended Three-Dimensional Analysis of Building System (ETABS) (version19) is a powerful software, used for building analysis and design. This software used for analysis purpose to this study. The study in this paper mainly strengthens on overturning moment and eccentricity of I-shaped fifteen storied building in both equivalent lateral force method and modal response spectrum method. The analyses are carried out as per ASCE7-16 and BNBC 2020 guidelines. It is observed that the overturning moment is approximately $11 \%$ greater in equivalent lateral force method than modal response spectrum method while in eccentricity approximately $2 \%$ greater in modal response spectrum method than equivalent lateral force method.

Keywords : ETABS, Analysis, Overturning moment, eccentricity, equivalent lateral fore method, modal response spectrum method.
\end{abstract}

\section{INTRODUCTION}

Lateral loads or earthquake loads are more sensitive to high-rise building. But when the eccentricity between mass centre and stiffness centre more the building induce irregularity and more vulnerable to seismic hazard [1]. The eccentricity depends on mass distribution and stiffness of each story on which centre of mass positioned. Due to eccentricity torsional effect induced. When earthquake shaking produce this torsional effect is more dangerous to building stability [2].

Building subjected to earthquake loading produce shear force in each story of building. These forces are produces overturning moment of the building. For rectangular regular shape building is less susceptible to overturning moment than I-shaped building.

The objectives of this work to study the eccentricity and overturning moment of I-shaped building in both equivalent lateral force method and modal response spectrum method.

\section{METHODS AND MATERIAL}

This work is based on software. A software name Extended Three-Dimensional Analysis of Building System (ETABS) version 19 used for this study. 15 (fifteen) storied residential building of I-shaped 
considered to study eccentricity and overturning moment. Equivalent Lateral Force Method and Modal Response Spectrum Analysis were used to perform for analysis. At first Equivalent Lateral Force Method was performed and then Modal Response Spectrum Analysis was performed. In analysis the materials properties considered as tabulated in table 2.1. The section properties tabulated in table 2.2. The load case and load combination tabulated in 2.3 and 2.4 respectively. For modal response spectrum analysis, the modes were selected 3 (three) times the number of stories. 2D plan, beam information, column information, shear wall position shown in figure 2.1, $2.2,2.3,2.4$ respectively. Also, 3D view shown in figure 2.5.

Table 2.1: materials properties

\begin{tabular}{|l|l|}
\hline \multicolumn{1}{|c|}{ Material type } & \multicolumn{1}{c|}{ Strength } \\
\hline $\begin{array}{l}\text { Concrete for column and shear } \\
\text { wall }\end{array}$ & $3500 \mathrm{psi}$ \\
\hline Concrete for beam and slab & $3000 \mathrm{psi}$ \\
\hline Rebar & 60 grade \\
\hline
\end{tabular}

Table 2.2: section properties

\begin{tabular}{|c|c|}
\hline Type & Size \\
\hline Column $1(\mathrm{C} 1)$ & $12^{\prime \prime} \times 25^{\prime \prime}$ \\
\hline Column $2(\mathrm{C} 1)$ & 12 ' $\times 27$ '" \\
\hline Column $3(\mathrm{C} 1)$ & 14 'x 30 '" \\
\hline Column $5(\mathrm{C} 1)$ & 12 'x18': \\
\hline Floor beam (B1-B5) & 10 'x 24 ': \\
\hline Grade beam & 12 ' $\times 24$ '" \\
\hline Shear wall & $10^{\prime \prime}$ \\
\hline Slab (shell thin) & $5.5^{\prime \prime}$ \\
\hline
\end{tabular}

Table 2.3: load cases

\begin{tabular}{|l|l|}
\hline Name & Type \\
\hline Dead & Linear Static \\
\hline Modal & Modal - Ritz \\
\hline WALL & Linear Static \\
\hline PW & Linear Static \\
\hline FF & Linear Static \\
\hline LIVE & Linear Static \\
\hline HOSPITAL LIVE & Linear Static \\
\hline STAIR LIVE & Linear Static \\
\hline ROOF LIVE & Linear Static \\
\hline LIFT DL & Linear Static \\
\hline LIFT LL & Linear Static \\
\hline OHWT & Linear Static \\
\hline EQX & Linear Static \\
\hline EQY & Linear Static \\
\hline WX & Linear Static \\
\hline WY & Linear Static \\
\hline RSX & Response spectrum \\
\hline RSY & Response spectrum \\
\hline
\end{tabular}

Table 2.4: Basic load combination

\begin{tabular}{|l|}
\hline $1.4 \mathrm{D}$ \\
\hline $1.2 \mathrm{D}+1.6 \mathrm{~L}$ \\
\hline $1.2 \mathrm{D}+1.0 \mathrm{~L}$ \\
\hline $1.2 \mathrm{D}+1.6 \mathrm{~W}+1.0 \mathrm{~L}$ \\
\hline $1.2 \mathrm{D}+1.0 \mathrm{E}+1.0 \mathrm{~L}$ \\
\hline $0.9 \mathrm{D}+1.6 \mathrm{~W}$ \\
\hline $0.9 \mathrm{D}+1.0 \mathrm{E}$ \\
\hline
\end{tabular}

Loads acting on the structure are dead load (DL), live load (LL), earthquake load, wind load. Self-weight consists of the weight of beam, column, slab and shear wall of the building. Wall Loads are Super Imposed Dead Load (SIDL). These loads are the loads of the brick walls which are located on the beams. Wall loads are calculated as per linear foot and applied on the model simultaneously as per wall positions.in this study wall load $0.5 \mathrm{k} / \mathrm{ft}$ applied. Partition Wall Loads are also Super Imposed Dead Load (SIDL). This load are the loads of the brick walls which are located on the slab panels. Partition wall loads are calculated as per square foot and applied on the model simultaneously as per wall positions. In this study 75psf applied Floor Finish Loads are also Super Imposed Dead Load (SIDL). These loads are the loads of the tiles, plasters on the slab panels. Floor finish loads are calculated assuming unit weight $145 \mathrm{pcf}$. and thickness of floor finish 1.5 inch.

Floor finish $=1.5 \mathrm{inX}(145 \mathrm{pcf})=18.125$ psf. $\approx 20$ psf. For Lift machine Load's shaft size Assumed 5'-4"X4'-8". From Public Works Department schedule, total load $=8$ tons $=17600 \mathrm{lb}$. Machine room floor area $=$ $7^{\prime} \times 66^{\prime}=42$ sq.ft. So, total lift machine load $=176000 / 42$ $=419.05$ psf. Portion of total load assumed as Dead load $=50 \%$ of total load $=209.52$ psf. Portion of total load assumed as Live load $=50 \%$ of total load $=209.52$ psf. Floor live load and roof live loads are applied separately on each floor and top of the floor. Stair live load applied in stair slab. Live load on the residential floor slabs $=42$ psf. Live load on the roof slab $=100$ psf. Live load on the stair $=100$ psf. Live load for hospital floors=100 psf. Wind load are applied in X and Y direction as per BNBC 2020, PART 6, Chapter 2. For seismic load: soil type: SC, Importance factor: 1 , Response reduction factor:7, Damping: $5 \%$, seismic zone: III as per BNBC 2020, PART 6, Chapter 2 [3]. 

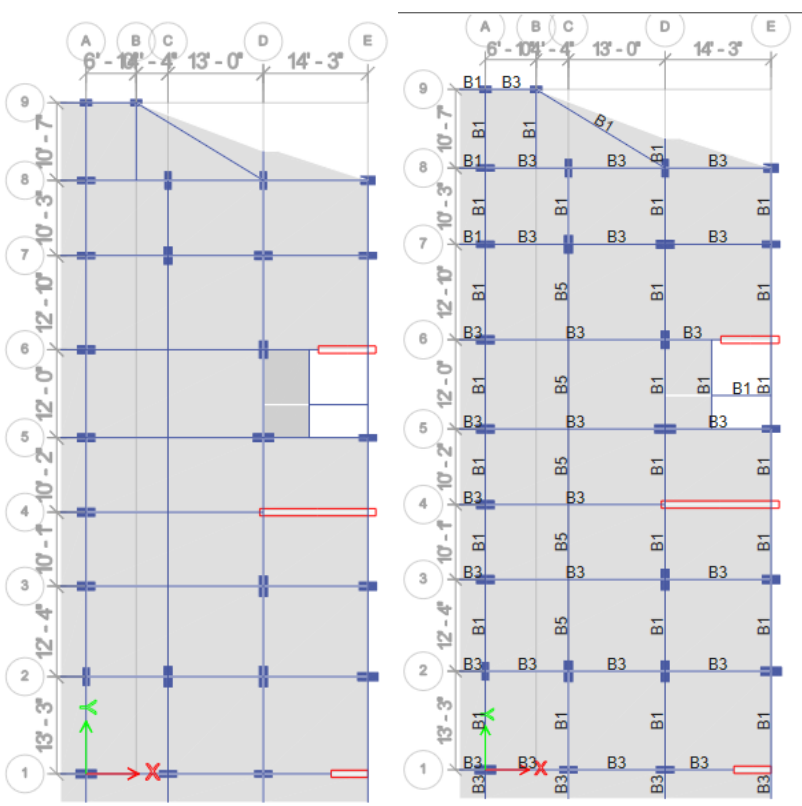

Fig.2.1 Plan of building

Fig.2.2 Beam information

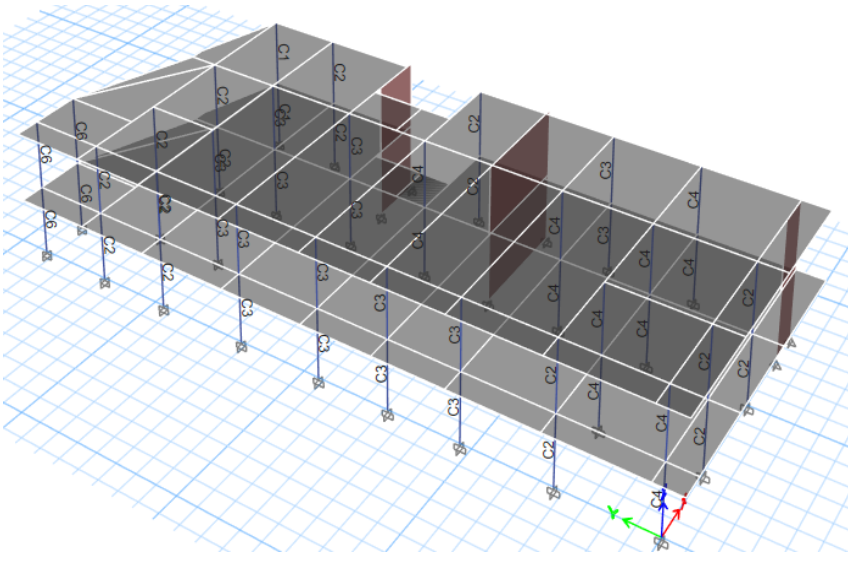

Fig2.3 Column information

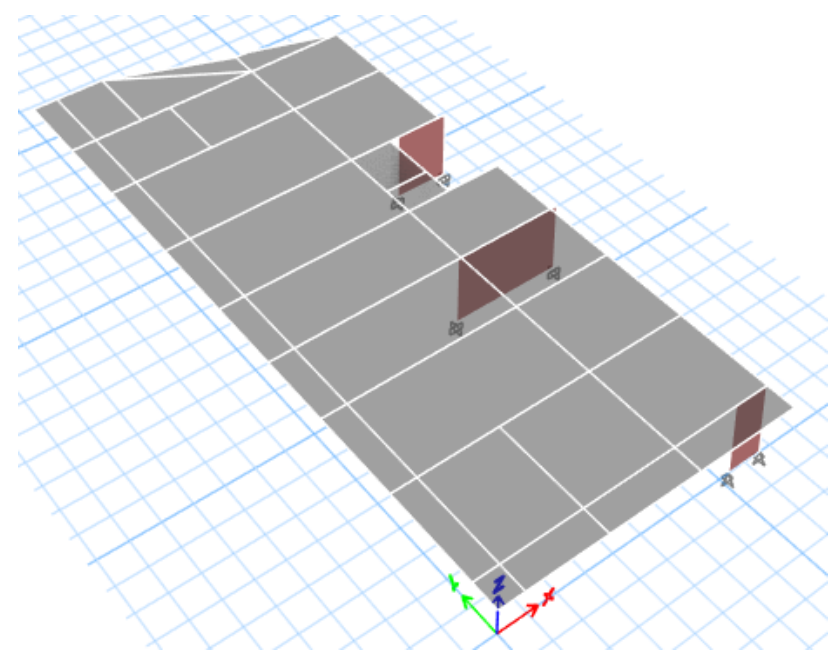

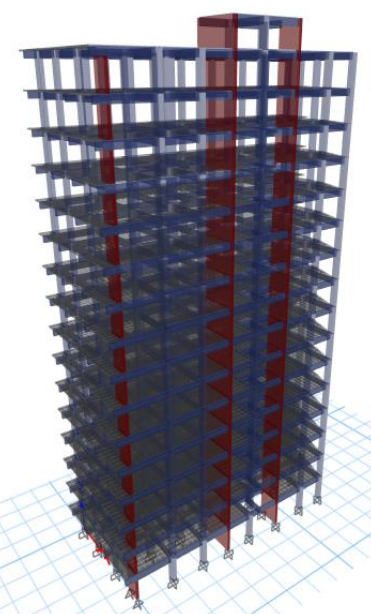

Fig.2.5 3D view of the building

\section{III.RESULTS AND DISCUSSION}

After completing the model, static force method and modal response analysis were running. The structure shall be designed to resist overturning effects caused by the seismic forces. At any story, the increment of overturning moment in the story under consideration shall be distributed to the various vertical force resisting elements in the same proportion as the distribution of the horizontal shears to those elements. The overturning moments at level $\mathrm{x}, \mathrm{Mx}$ determined as follows:

$$
M_{x}=\sum_{i=x}^{n} F_{i}\left(h_{i}-h_{x}\right)
$$

where,

$\mathrm{F}=$ Portion of the seismic base shear, $\mathrm{V}$

induced at level $\mathrm{i}$

$h_{i}, h_{x}=$ Height from the base to level $i$ or $x$.

Overturning moment for earthquake load for equivalent lateral force method in $\mathrm{x}$ direction was found as 63718 kip- $\mathrm{ft}$ which calculation shown in the table 3.1, where in modal response spectrum analysis was found as $56308 \mathrm{kip}-\mathrm{ft}$. shown in table 3.2. In Y direction overturning moment was found as 63318 kip-ft in equivalent lateral force method shown in table 3.3 where in response spectrum analysis was found as $56442 \mathrm{kip}-\mathrm{ft}$ shown in table 3.4.

Fig2.4 Shear wall position 
Table3.1: The calculation of overturning moment for earthquake load in $\mathrm{x}$ direction in equivalent lateral force method

\begin{tabular}{|c|c|c|c|c|c|}
\hline Level & $\begin{array}{c}\text { Story } \\
\text { Ht }\end{array}$ & $\begin{array}{c}\text { Elev } \\
\cdot\end{array}$ & $\begin{array}{c}\text { ETAB } \\
\text { S } \\
\text { Cum. } \\
\text { Story } \\
\text { Force/ } \\
\text { Shear } \\
(\mathbf{V x}) \\
\end{array}$ & $\begin{array}{c}\text { Individual } \\
\text { Story Force/ } \\
\text { Shear (Vx) }\end{array}$ & $\begin{array}{c}\text { Overturning } \\
\text { Moment (OM) }\end{array}$ \\
\hline $\begin{array}{c}\text { ROO } \\
\text { F }\end{array}$ & 10 & 160 & 58.641 & 58.641 & 9382.56 \\
\hline F14 & 10 & 150 & $\begin{array}{c}128.14 \\
1 \\
\end{array}$ & 69.5 & 11120 \\
\hline F13 & 10 & 140 & $\begin{array}{c}190.72 \\
2 \\
\end{array}$ & 62.581 & 9387.15 \\
\hline F12 & 10 & 130 & $\begin{array}{c}246.63 \\
6\end{array}$ & 55.914 & 7827.96 \\
\hline F11 & 10 & 120 & $\begin{array}{c}296.14 \\
5 \\
\end{array}$ & 49.509 & 6436.17 \\
\hline F10 & 10 & 110 & 339.52 & 43.375 & 5205 \\
\hline F9 & 10 & 100 & $\begin{array}{c}377.04 \\
6\end{array}$ & 37.526 & 4127.86 \\
\hline F8 & 10 & 90 & $\begin{array}{c}409.01 \\
8\end{array}$ & 31.972 & 3197.2 \\
\hline F7 & 10 & 80 & 435.75 & 26.732 & 2405.88 \\
\hline F6 & 10 & 70 & $\begin{array}{c}457.57 \\
1\end{array}$ & 21.821 & 1745.68 \\
\hline F5 & 10 & 60 & $\begin{array}{c}474.83 \\
4\end{array}$ & 17.263 & 1208.41 \\
\hline F4 & 10 & 50 & $\begin{array}{c}487.91 \\
8\end{array}$ & 13.084 & 785.04 \\
\hline F3 & 10 & 40 & $\begin{array}{c}497.23 \\
9\end{array}$ & 9.321 & 466.05 \\
\hline $\mathrm{F} 2$ & 10 & 30 & $\begin{array}{c}504.29 \\
6 \\
\end{array}$ & 7.057 & 282.28 \\
\hline F1 & 10 & 20 & $\begin{array}{c}508.10 \\
2\end{array}$ & 3.806 & 114.18 \\
\hline GF & 10 & 10 & $\begin{array}{c}509.45 \\
2 \\
\end{array}$ & 1.35 & 27 \\
\hline \multicolumn{5}{|c|}{\begin{tabular}{|c|c} 
& Base Shear= \\
509
\end{tabular}} & $\begin{array}{c}\text { Total OM= } \\
63718\end{array}$ \\
\hline
\end{tabular}

Table : 3.2 The calculation of overturning moment for earthquake load in $\mathrm{x}$ direction in modal response spectrum analysis method

\begin{tabular}{|c|c|c|c|c|c|}
\hline Level & $\begin{array}{c}\text { Story } \\
\text { Ht }\end{array}$ & Elev. & $\begin{array}{c}\text { ETABS } \\
\text { Cum. } \\
\text { Story } \\
\text { force/ } \\
\text { Shear } \\
\text { (Vx) }\end{array}$ & $\begin{array}{c}\text { Individual } \\
\text { Story } \\
\text { Force/ } \\
\text { Shear } \\
\text { (Vx) }\end{array}$ & $\begin{array}{c}\text { Overturning } \\
\text { Moment } \\
\text { (OM) }\end{array}$ \\
\hline ROOF & 10 & 160 & 21.438 & 21.438 & 3430.08 \\
\hline F14 & 10 & 150 & 98.327 & 76.889 & 12302.24 \\
\hline F13 & 10 & 140 & 169.273 & 70.946 & 10641.9 \\
\hline F12 & 10 & 130 & 219.589 & 50.316 & 7044.24 \\
\hline F11 & 10 & 120 & 256.572 & 36.983 & 4807.79 \\
\hline F10 & 10 & 110 & 284.918 & 28.346 & 3401.52 \\
\hline F9 & 10 & 100 & 307.599 & 22.681 & 2494.91 \\
\hline F8 & 10 & 90 & 327.239 & 19.64 & 1964 \\
\hline F7 & 10 & 80 & 346.589 & 19.35 & 1741.5 \\
\hline F6 & 10 & 70 & 368.074 & 21.485 & 1718.8 \\
\hline F5 & 10 & 60 & 392.315 & 24.241 & 1696.87 \\
\hline F4 & 10 & 50 & 418.372 & 26.057 & 1563.42 \\
\hline F3 & 10 & 40 & 444.605 & 26.233 & 1311.65 \\
\hline F2 & 10 & 30 & 469.632 & 25.027 & 1001.08 \\
\hline F1 & 10 & 20 & 495.992 & 26.36 & 790.8 \\
\hline GF & 10 & 10 & 515.853 & 19.861 & 397.22 \\
\hline & & & & Base Shear & Total OM \\
& & & & 56308 \\
\hline
\end{tabular}

Table3.3: The calculation of overturning moment for earthquake load

in $\mathrm{Y}$ direction in equivalent lateral force method

\begin{tabular}{|c|c|c|c|c|c|}
\hline Level & $\begin{array}{c}\text { Story } \\
\text { Ht }\end{array}$ & Elev. & $\begin{array}{c}\text { ETABS } \\
\text { Cum. } \\
\text { Story } \\
\text { Force } \\
\text { /Shear } \\
\text { (Vy) }\end{array}$ & $\begin{array}{c}\text { Indivitual } \\
\text { Story } \\
\text { Force } \\
\text { /Shear } \\
\text { (Vy) }\end{array}$ & $\begin{array}{c}\text { Overturning } \\
\text { Moment } \\
(\mathbf{O M})\end{array}$ \\
\hline ROOF & 10 & 160 & 58.641 & 58.641 & 9382.56 \\
\hline F14 & 10 & 150 & 128.141 & 69.5 & 11120 \\
\hline F13 & 10 & 140 & 190.722 & 62.581 & 9387.15 \\
\hline F12 & 10 & 130 & 246.636 & 55.914 & 7827.96 \\
\hline F11 & 10 & 120 & 296.145 & 49.509 & 6436.17 \\
\hline F10 & 10 & 110 & 339.52 & 43.375 & 5205 \\
\hline F9 & 10 & 100 & 377.046 & 37.526 & 4127.86 \\
\hline F8 & 10 & 90 & 409.018 & 31.972 & 3197.2 \\
\hline F7 & 10 & 80 & 435.75 & 26.732 & 2405.88 \\
\hline F6 & 10 & 70 & 457.571 & 21.821 & 1745.68 \\
\hline F5 & 10 & 60 & 474.834 & 17.263 & 1208.41 \\
\hline F4 & 10 & 50 & 487.918 & 13.084 & 785.04 \\
\hline F3 & 10 & 40 & 497.239 & 9.321 & 466.05 \\
\hline F2 & 10 & 30 & 504.296 & 7.057 & 282.28 \\
\hline F1 & 10 & 20 & 508.102 & 3.806 & 114.18 \\
\hline GF & 10 & 10 & 509.452 & 1.35 & 27 \\
\hline & & & & $\begin{array}{c}\text { Base Shear } \\
=509\end{array}$ & $\begin{array}{c}\text { Total OM } \\
=63718\end{array}$ \\
\hline & & & & &
\end{tabular}


Table : 3.4 The calculation of overturning moment for earthquake load in $\mathrm{Y}$ direction in modal response spectrum analysis method

\begin{tabular}{|c|c|c|c|c|c|}
\hline Level & $\begin{array}{c}\text { Story } \\
\text { Ht }\end{array}$ & Elev. & $\begin{array}{c}\text { ETABS } \\
\text { Cum. } \\
\text { Story } \\
\text { Force/ } \\
\text { Shear } \\
\text { (Vy) }\end{array}$ & $\begin{array}{c}\text { Indivitual } \\
\text { Story } \\
\text { Force/Shear } \\
\text { (Vy) }\end{array}$ & $\begin{array}{c}\text { Overturning } \\
\text { Moment } \\
\text { (OM) }\end{array}$ \\
\hline ROOF & 10 & 160 & 17.61 & 17.61 & 2817.6 \\
\hline F14 & 10 & 150 & 84.88 & 67.27 & 10763.2 \\
\hline F13 & 10 & 140 & 157.717 & 72.837 & 10925.55 \\
\hline F12 & 10 & 130 & 215.638 & 57.921 & 8108.94 \\
\hline F11 & 10 & 120 & 260.382 & 44.744 & 5816.72 \\
\hline F10 & 10 & 110 & 294.161 & 33.779 & 4053.48 \\
\hline F9 & 10 & 100 & 319.567 & 25.406 & 2794.66 \\
\hline F8 & 10 & 90 & 339.365 & 19.798 & 1979.8 \\
\hline F7 & 10 & 80 & 356.345 & 16.98 & 1528.2 \\
\hline F6 & 10 & 70 & 373.198 & 16.853 & 1348.24 \\
\hline F5 & 10 & 60 & 392.049 & 18.851 & 1319.57 \\
\hline F4 & 10 & 50 & 413.953 & 21.904 & 1314.24 \\
\hline F3 & 10 & 40 & 438.725 & 24.772 & 1238.6 \\
\hline F2 & 10 & 30 & 464.924 & 26.199 & 1047.96 \\
\hline F1 & 10 & 20 & 494.811 & 29.887 & 896.61 \\
\hline GF & 10 & 10 & 519.267 & 24.456 & 489.12 \\
\hline FF & & & & Base Shear & Total OM \\
& & & & 56442 \\
\hline FI & the & & & \\
\hline
\end{tabular}

From the above tables it observed that the overturning moments are higher in both direction in equivalent lateral force method than response spectrum method. Overturning moment in top floor is high cause the story shear is high and multiply with highest distance from bottom to top for equivalent lateral force method while in response spectrum method the story shear is so less.

From figure 3.1 and 3.2 it shown that overall overturning moment in equivalent lateral force method is $11.63 \%$ greater than the response spectrum method in $\mathrm{X}$ direction and $11.42 \%$ in $\mathrm{Y}$ direction.

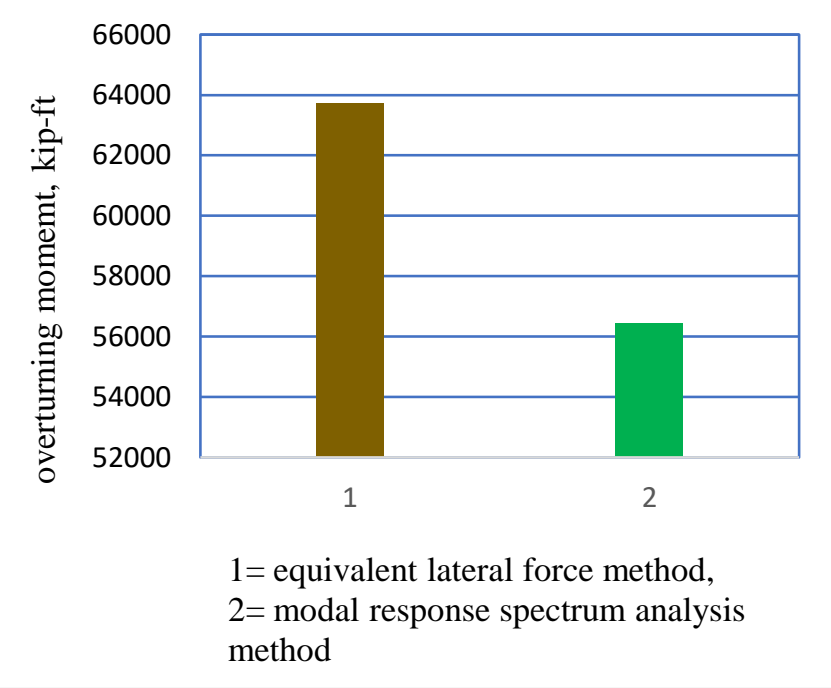

Fig. 3.1: variation of overturning moment in $\mathrm{X}$ direction

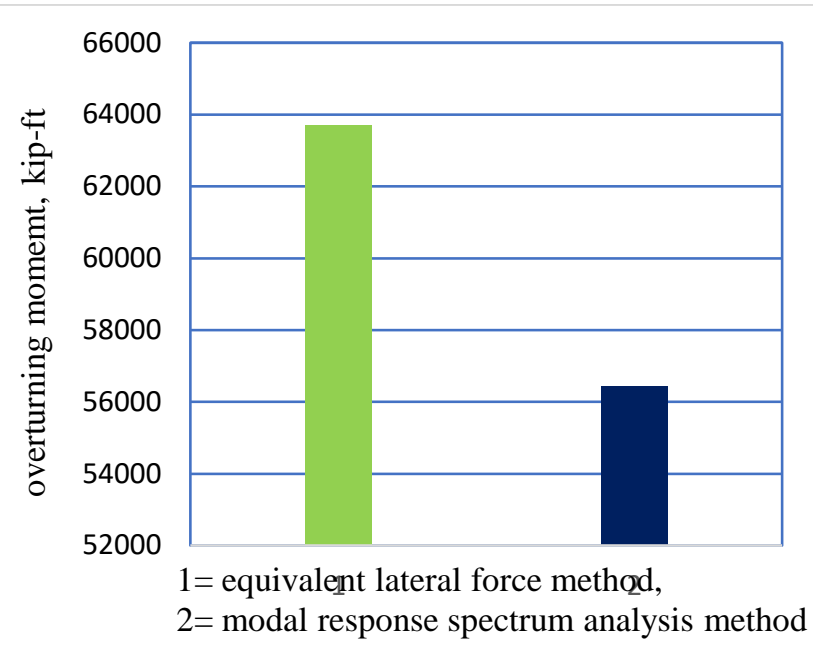

Fig. 3.2 : variation of overturning moment in $\mathrm{Y}$ direction

The eccentricity between the centre of mass and the geometric centroid of the building at that level shall not exceed $15 \%$ of the overall building width along each principal axis considered at each level [4]. In table 3.5 and table 3.6 eccentricity calculations are shown.

Table 3.5: The calculation of eccentricity in equivalent lateral force method

\begin{tabular}{|c|c|c|c|c|c|c|}
\hline \multirow{2}{*}{ Story } & \multicolumn{2}{|c|}{ C.M } & \multicolumn{2}{c|}{ C.R } & \multicolumn{2}{c|}{ Eccentricity } \\
\cline { 2 - 7 } & $\mathbf{x}_{\mathbf{m}}$ & $\mathbf{y}_{\mathbf{m}}$ & $\mathbf{X C R}$ & $\mathbf{Y C R}$ & $\mathbf{e}_{\mathbf{x}}$ & $\mathbf{e}_{\mathbf{y}}$ \\
\hline ROOF & 18.6198 & 41.5682 & 19.80 & 38.58 & 1.18 & 2.99 \\
\hline F14 & 17.0505 & 41.996 & 19.73 & 38.61 & 2.68 & 3.39 \\
\hline F13 & 17.0505 & 41.996 & 19.67 & 38.67 & 2.62 & 3.33 \\
\hline F12 & 17.0505 & 41.996 & 19.61 & 38.73 & 2.55 & 3.26 \\
\hline F11 & 17.0505 & 41.996 & 19.55 & 38.80 & 2.50 & 3.20 \\
\hline F10 & 17.0505 & 41.996 & 19.49 & 38.85 & 2.44 & 3.15 \\
\hline F9 & 17.0505 & 41.996 & 19.44 & 38.89 & 2.39 & 3.11 \\
\hline F8 & 17.0505 & 41.996 & 19.38 & 38.92 & 2.33 & 3.08 \\
\hline F7 & 17.0505 & 41.996 & 19.34 & 38.93 & 2.29 & 3.07 \\
\hline F6 & 17.0505 & 41.996 & 19.29 & 38.91 & 2.24 & 3.09 \\
\hline F5 & 17.0505 & 41.996 & 19.24 & 38.87 & 2.19 & 3.13 \\
\hline F4 & 17.0505 & 41.996 & 19.20 & 38.79 & 2.15 & 3.21 \\
\hline F3 & 17.0505 & 41.996 & 19.15 & 38.67 & 2.10 & 3.33 \\
\hline F2 & 16.9067 & 42.1302 & 19.08 & 38.49 & 2.17 & 3.64 \\
\hline F1 & 16.9027 & 42.1098 & 18.96 & 38.33 & 2.06 & 3.78 \\
\hline GF & 16.9805 & 42.2318 & 18.67 & 37.94 & 1.69 & 4.29 \\
\hline
\end{tabular}


Table 3.6: The calculation of eccentricity in modal response spectrum method

\begin{tabular}{|c|c|c|c|c|c|c|}
\hline \multirow{2}{*}{ Story } & \multicolumn{2}{|c|}{ C.M } & \multicolumn{2}{c|}{ C.R } & \multicolumn{2}{|c|}{ Eccentricity } \\
\cline { 2 - 7 } & $\mathbf{x}_{\mathbf{m}}$ & $\mathbf{y}_{\mathbf{m}}$ & $\mathbf{X C R}$ & $\mathbf{Y C R}$ & $\mathbf{e}_{\mathbf{x}}$ & $\mathbf{~}_{\mathbf{y}}$ \\
\hline ROOF & 18.6198 & 41.5682 & 19.86 & 38.47 & 1.24 & 3.10 \\
\hline F14 & 17.0505 & 41.996 & 19.79 & 38.50 & 2.74 & 3.49 \\
\hline F13 & 17.0505 & 41.996 & 19.73 & 38.56 & 2.68 & 3.43 \\
\hline F12 & 17.0505 & 41.996 & 19.68 & 38.63 & 2.63 & 3.37 \\
\hline F11 & 17.0505 & 41.996 & 19.62 & 38.69 & 2.57 & 3.31 \\
\hline F10 & 17.0505 & 41.996 & 19.57 & 38.75 & 2.52 & 3.25 \\
\hline F9 & 17.0505 & 41.996 & 19.51 & 38.79 & 2.46 & 3.20 \\
\hline F8 & 17.0505 & 41.996 & 19.46 & 38.82 & 2.41 & 3.17 \\
\hline F7 & 17.0505 & 41.996 & 19.41 & 38.84 & 2.36 & 3.16 \\
\hline F6 & 17.0505 & 41.996 & 19.36 & 38.83 & 2.31 & 3.16 \\
\hline F5 & 17.0505 & 41.996 & 19.31 & 38.80 & 2.26 & 3.20 \\
\hline F4 & 17.0505 & 41.996 & 19.26 & 38.73 & 2.21 & 3.27 \\
\hline F3 & 17.0505 & 41.996 & 19.21 & 38.62 & 2.15 & 3.37 \\
\hline F2 & 16.9067 & 42.1302 & 19.12 & 38.46 & 2.22 & 3.67 \\
\hline F1 & 16.9027 & 42.1098 & 18.99 & 38.31 & 2.09 & 3.80 \\
\hline GF & 16.9805 & 42.2318 & 18.69 & 37.93 & 1.71 & 4.30 \\
\hline
\end{tabular}

From the table 3.7 and table 3.8 it shown that the eccentricity value increased $2.7 \%$ average in modal response spectrum method than equivalent lateral force method in $\mathrm{X}$ direction and $2.28 \%$ in $\mathrm{Y}$ direction.

Table 3.7: percent difference in eccentricity in $\mathrm{X}$ direction

\begin{tabular}{|c|c|c|c|}
\hline Level & $\begin{array}{c}\text { e } \\
\text { equivalent } \\
\text { lateral force } \\
\text { method }\end{array}$ & $\begin{array}{c}\mathrm{e}_{\mathrm{x}}, \\
\text { modal response } \\
\text { spectrum method }\end{array}$ & \% Difference \\
\hline ROOF & 1.18 & 1.24 & 4.83871 \\
\hline F14 & 2.68 & 2.74 & 2.189781 \\
\hline F13 & 2.62 & 2.68 & 2.238806 \\
\hline F12 & 2.55 & 2.63 & 3.041825 \\
\hline F11 & 2.5 & 2.57 & 2.723735 \\
\hline F10 & 2.44 & 2.52 & 3.174603 \\
\hline F9 & 2.39 & 2.46 & 2.845528 \\
\hline F8 & 2.33 & 2.41 & 3.319502 \\
\hline F7 & 2.29 & 2.36 & 2.966102 \\
\hline F6 & 2.24 & 2.31 & 3.030303 \\
\hline F5 & 2.19 & 2.26 & 3.097345 \\
\hline F4 & 2.15 & 2.21 & 2.714932 \\
\hline F3 & 2.1 & 2.15 & 2.325581 \\
\hline F2 & 2.17 & 2.22 & 2.252252 \\
\hline F1 & 2.06 & 2.09 & 1.435407 \\
\hline GF & 1.69 & 1.71 & 1.169591 \\
\hline
\end{tabular}

Table 3.8: percent difference in eccentricity in $\mathrm{Y}$ direction

\begin{tabular}{|c|c|c|c|}
\hline level & $\begin{array}{c}\text { ey, equivalent } \\
\text { lateral force } \\
\text { method }\end{array}$ & $\begin{array}{c}\text { ey, } \\
\text { modal response } \\
\text { spectrum } \\
\text { method }\end{array}$ & \% Difference \\
\hline ROOF & 2.99 & 3.1 & 3.548387 \\
\hline F14 & 3.39 & 3.49 & 2.86533 \\
\hline F13 & 3.33 & 3.43 & 2.915452 \\
\hline F12 & 3.26 & 3.37 & 3.264095 \\
\hline F11 & 3.2 & 3.31 & 3.323263 \\
\hline F10 & 3.15 & 3.25 & 3.076923 \\
\hline F9 & 3.11 & 3.2 & 2.8125 \\
\hline F8 & 3.08 & 3.17 & 2.839117 \\
\hline F7 & 3.07 & 3.16 & 2.848101 \\
\hline F6 & 3.09 & 3.16 & 2.21519 \\
\hline F5 & 3.13 & 3.2 & 2.1875 \\
\hline F4 & 3.21 & 3.27 & 1.834862 \\
\hline F3 & 3.33 & 3.37 & 1.186944 \\
\hline F2 & 3.64 & 3.67 & 0.817439 \\
\hline F1 & 3.78 & 3.8 & 0.526316 \\
\hline GF & 4.29 & 4.3 & 0.232558 \\
\hline
\end{tabular}

\section{IV.CONCLUSION}

Equivalent lateral force method gives higher value for overturning moment especially in high-rise building which is more conservative than response spectrum method. Equivalent lateral force method gives lower value for eccentricity than response spectrum method. High value of overturning moment is more important in I-shaped building for stability of the building. During earthquake response overturning moment affect the foundation of the building. However as for equivalent lateral force method is more conservative response spectrum method can be used for high rise building. Though eccentricity is greater in response spectrum method but the values are less difference in percentage in both analysis method.

Conclusion are based on findings of the study; further study should be done using different shape of building with plan irregularity and height. Also, torsional moment effect due to eccentricity should be studied in further.

\section{REFERENCES}

[1]. Devikashree M L, B.S Jayashankara babu. 2017. A Study on Effect of Eccentricity in RC Frame Structures, International Journal of Innovative Research in Science, Engineering and 
Technology, Vol. 6, Issue 6, June 2017, ISSN(Online): 2319-8753

[2]. Atif Zakaria, M. Shiva Rama Krishna, T.G.N.C.Vamsi Krishna, Mirza Mahaboob Baig. 2019. Effects of the Accidental Eccentricity on Regular and Irregular Buildings, International Journal of Innovative Technology and Exploring Engineering (IJTEE), Volume-8 Issue-11, September 2019, ISSN: 2278-3075

[3]. BNBC (part 6, chapter 2) 2020, Bangladesh National Building Code, Bangladesh Government Press, Dhaka, Bangladesh.

[4]. ASCE/SEI/7-16 (appendix D), Minimum Design Loads and Associated Criteria for Buildings and other Structures, American Society of Civil Engineers.

\section{Cite this article as :}

M. T. Hasan, M. L. Hossain, "Comparative Study on Overturning Moment and Eccentricity of A I-Shaped Building by Equivalent Lateral Force and Modal Response Spectrum Method Using ETABS Software", International Journal of Scientific Research in Science and Technology (IJSRST), Online ISSN : 2395-602X, Print ISSN : 2395-6011, Volume 8 Issue 4, pp. 173-179, July-August 2021. Available at doi : https://doi.org/10.32628/IJSRST218262

Journal URL : https://ijsrst.com/IJSRST218262 